\title{
Optimized wide-field survey telescope using adaptive optics
}

Bernhard Rainer Brandl, Richard G. Dekany, Riccardo Giovanelli

Bernhard Rainer Brandl, Richard G. Dekany, Riccardo Giovanelli, "Optimized wide-field survey telescope using adaptive optics," Proc. SPIE 4836, Survey and Other Telescope Technologies and Discoveries, (24 December 2002); doi: $10.1117 / 12.457295$

SPIE Event: Astronomical Telescopes and Instrumentation, 2002, Waikoloa, Hawai'i, United States 


\title{
An Optimized Wide-Field Survey Telescope Using Adaptive Optics
}

\author{
Bernhard R. Brandl ${ }^{a}$, Richard Dekany ${ }^{b}$, and Riccardo Giovenelli ${ }^{a}$ \\ ${ }^{a}$ Center for Radiophysics and Space Research, Cornell University, Ithaca, NY 14853 \\ ${ }^{b}$ Department of Astronomy, Caltech, Pasadena, CA 91125
}

\begin{abstract}
We describe a new technique for ground-based telescopic surveys that can deliver a wide field of view and nearly diffraction-limited image quality. We discuss a very low cost, yet sensitive and efficient, concept to perform science previously considered from space.

For ground-based telescopes with small $\mathrm{D} / \mathrm{r}_{0}$ (aperture over turbulence cell diameter) a significant improvement in point source sensitivity can be achieved with tip-tilt correction only. However, the solid angle over which image motion is constant is typically less than an arcminute. To achieve tip-tilt correction over a larger field we propose to use a high order adaptive optics system where one pupil sub-aperture now corresponds to one isokinetic patch. The high order deformable mirror is conjugated to an atmospheric height where the tip-tilt "beams" separate from each other while the overall tip-tilt can be taken out with a tip-tilt secondary mirror conjugated to low height.
\end{abstract}

One source per square arcminute with $\mathrm{V} \leq 18^{\mathrm{m}}$ is required for the determination of the image motion, allowing a sky coverage of more than $50 \%$. The improvement over seeing limited observations is maximal at $\mathrm{D} / \mathrm{r}_{0} \approx 4$ with a $\mathrm{S} / \mathrm{N}$ improvement of a factor of four. An inexpensive system with 500 actuators can correct a field of view of $0.4 \times 0.4$ $\mathrm{deg}^{2}$. It is thus well-suited for searches of point sources, e.g. high-z SN Ia or other transient phenomena.

Keywords: survey telescope, adaptive optics, tip-tilt correction, wide field-of-view, CCD array camera, supernovae

\section{SCIENTIFIC MOTIVATION}

Relatively small, seeing limited telescopes on the ground have enabled a wealth of extremely useful surveys at many optical and near-IR wavelengths. Because of the seeing and small aperture their sensitivity and spatial resolution is rather limited. On the other hand, new large-aperture telescopes using adaptive optics (AO) have a relatively small AO-corrected field-of-view (FOV) and are rarely being used for time-consuming large area surveys.

A small and affordable telescope with quasi-diffraction limited resolution - and hence significantly increased sensitivity to point sources - over a wide field of view would combine the advantages of both concepts and fill the gap. The proposed instrument described in this paper would enable, besides many other scientific projects:

- Time resolved $(\approx 50 \mathrm{~Hz})$ imaging survey of/for transient phenomena

- Searches for moving targets (e.g., asteroids)

- Targets of unknown position that require high angular resolution (e.g., compact gravitational lenses)

- Searches for supernovae (SNe)

Of these, the last item may currently attract the most interest. As standard candles to measure the expansion rate of the Universe and hence the amount of dark energy and the ultimate fate of the Universe, SNe of type Ia have become of great interest to cosmologist. However, only a small sample of less than a hundred $\mathrm{SNe}$ at relatively low redshifts has been studied so far and larger samples are needed. Table 1 lists supernova rates for a given FOV and redshift interval. For a FOV of e.g. $0.35 \times 0.35 \mathrm{deg}^{2}$ (please note that the FOV size is just an example and not given by technical limitations of the proposed concept), an integration time of 30 minutes per fields, 10 fields per night, and a total of 50 fields per year (i.e., reobserving each field every 5 days) we expect a total of about $500 \mathrm{SNe} /$ year out to a redshift of one. 


\begin{tabular}{|c|c|c|c|}
\hline Redshift & Peak $[\mu \mathrm{m}]$ & Peak $[\mathrm{mag}]$ & $\Sigma \mathrm{SNe} / \mathrm{FOV} / \mathrm{yr}$ \\
\hline 0.2 & 0.53 & 20.6 & 0.36 \\
\hline 0.4 & 0.62 & 22.3 & 1.62 \\
\hline 0.6 & 0.70 & 23.4 & 3.81 \\
\hline 0.8 & 0.79 & 24.2 & 6.73 \\
\hline 1.0 & 0.88 & 24.8 & 10.1 \\
\hline
\end{tabular}

Table 1: Expected supernova rates and related parameters (based on information provided on the SNAP website ${ }^{1}$ ). 'Peak' refers to the wavelength and magnitude of the peak emission of the redshifted light curve. The last column is the total number of supernovae in a $0.35 \times 0.35 \mathrm{deg}^{2}$ field-of-view at a redshift between zero and the given value per year. Note that the FOV size is just an example without technical limitation.

\section{SPECKLES AND TIP-TILT CORRECTION}

In simple terms, the atmosphere above the telescope can be described by patches of constant temperature (and hence density) over which the incoming wavefront (for a given wavelength) will be flat, the so-called Fried cells with diameter $\mathrm{r}_{0}$. If there were only one such cell across the full aperture the image would be diffraction limited and experience only image motion (tip-tilt). The light passing through different cells across the aperture interferes and causes a time- and spatially variable speckle pattern whose time-average is well known as astronomical seeing. The speckles may have different intensities but still contain the diffraction-limited information of the source. Generally, the worse the seeing and the larger the aperture D of the telescope the more speckles there will be, washing out the diffraction limited information on time scales of milliseconds.

However, if the number of Fried cells over the full aperture $\left(D / r_{0}\right)$ is small, and if it is possible to follow the brightest speckle in real-time, one can preserve a large fraction of the diffraction-limited information. This technique of "peak tracking", also called shift-and-add, yields a high gain over seeing limited observations, but requires a complete sampling of the speckle pattern. On the other hand, tip-tilt systems that follow the speckle pattern over time using a flat tip-tilt mirror can work on much fainter guide stars since they only need to determine the centroid using a quad-cell detector. $87 \%$ of the power lies in the tip-tilt term alone ${ }^{2}$, and hence one would expect tip-tilt correction to have a significant effect on the image quality on small telescopes or under excellent seeing. For large telescopes (large $\left.\mathrm{D} / \mathrm{r}_{0}\right)$ with hundreds of speckles the centroid of the speckle distribution does not wander around and the resulting improvement is only marginal; in that case a full AO system is needed to "combine the speckles", i.e. flatten the disturbed wavefront. For small $\mathrm{D} / \mathrm{r}_{0}$ Table 2 shows that centroid tracking using a tip-tilt system can deliver a great improvement over seeing limited imaging. Kaiser et al. ${ }^{3}$ have done simulations for $\lambda=0.8 \mu \mathrm{m}$ and $\mathrm{r}_{0}=0.4 \mathrm{~m}$ and found that the distribution of Strehl ratio gain peaks at $\mathrm{D} / \mathrm{r}_{0} \approx 4$ (see Figure 1).

\begin{tabular}{|c|c|c|c|}
\hline $\mathrm{D} / \mathrm{r}_{0}$ & $\mathrm{SR}$ (centroid) & $\mathrm{SR}$ (seeing) & SR gain \\
\hline 3.45 & 0.32 & 0.07 & 4.6 \\
\hline 4.22 & 0.21 & 0.05 & 4.2 \\
\hline 5.43 & 0.11 & 0.03 & 3.7 \\
\hline
\end{tabular}

Table 2: Relative gain of tip-tilt tracking over seeing limited observations in terms of Strehl ratio for various cases of $D / r_{0}$. Values taken from Christou ${ }^{4}$.

Figure 1: The gain in Strehl ratio as a function of $\mathrm{D} / \mathrm{r}_{0}$ for $\lambda=0.8 \mu \mathrm{m}$ and $\mathbf{r}_{0}=0.4 \mathrm{~m}$ (Kaiser et al. $^{3}$ ). The pronounced peak is at $D / r_{0} \approx 4$.

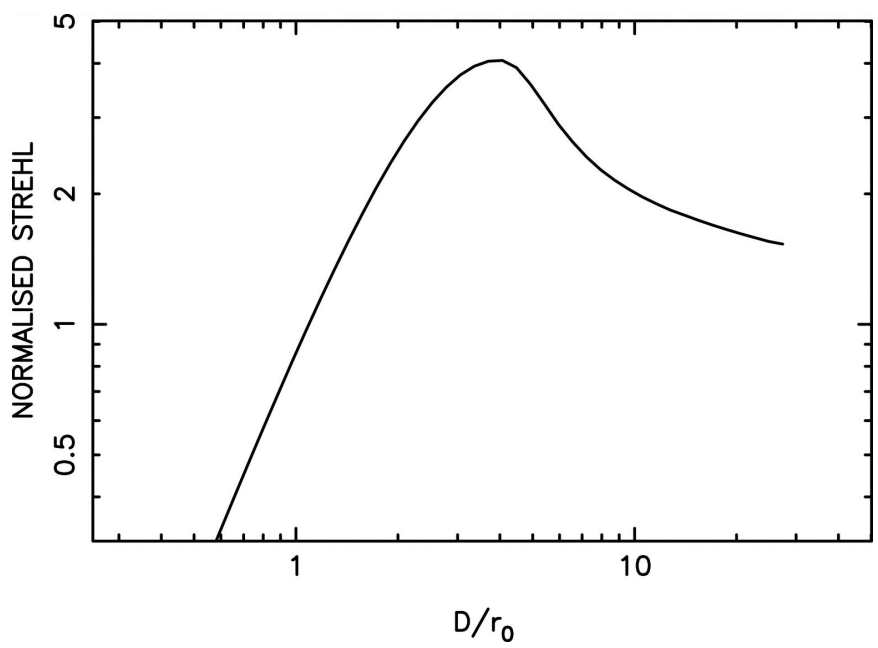


Note, that a gain of 4 means that the same signal-to-noise $(\mathrm{S} / \mathrm{N})$ as from a seeing limited telescope with four times larger aperture is achieved, since for background limited performance (BLIP) $\mathrm{S} / \mathrm{N} \propto \mathrm{D} /$ source-diameter. The assumption of BLIP even for short wavelengths is reasonable, at least for $\mathrm{SNe}$ searches where the light from the host galaxy increases the "background" flux. On the other hand, at $\mathrm{D} / \mathrm{r}_{0} \approx 4$ one would expect some of the light in fainter speckles not being concentrated into the diffraction limited PSF but spread out into a wider halo. Figure 2 shows a comparison between seeing limited PSF, tip-tilt PSF for $\mathrm{D} / \mathrm{r}_{0} \approx 4$, and diffraction limited PSF.
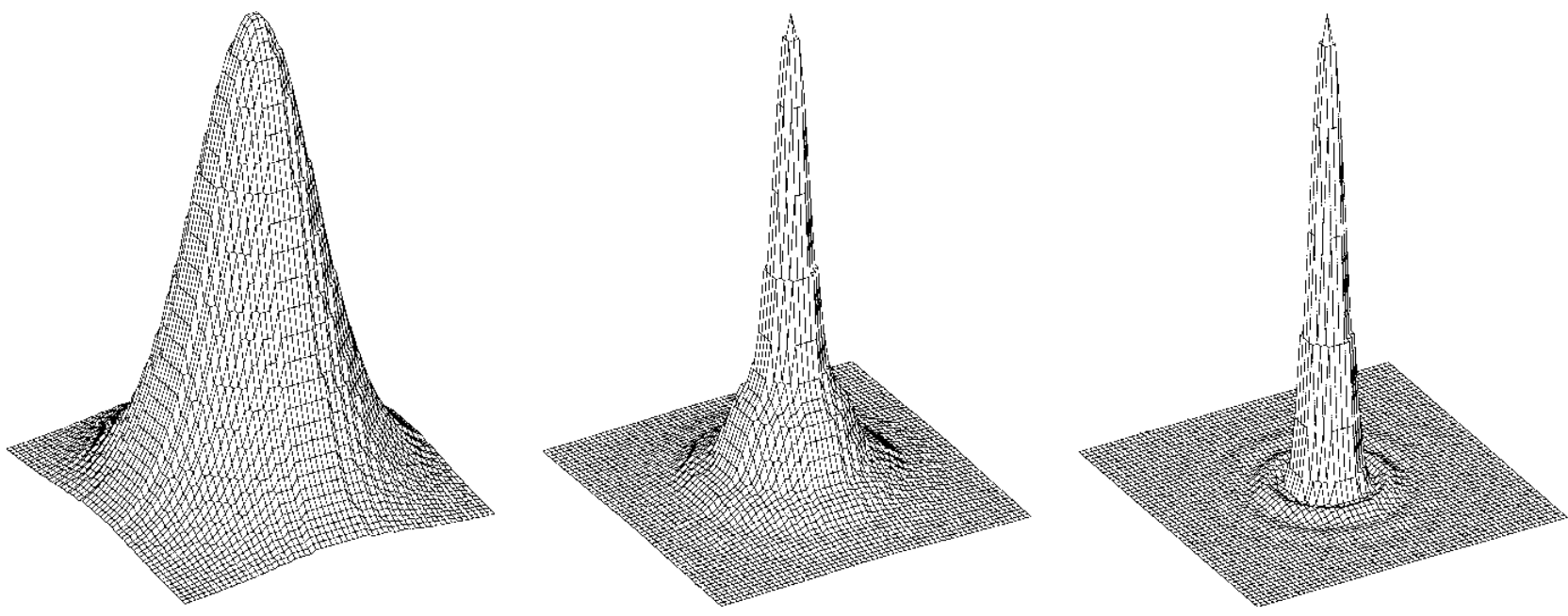

Figure 2: Comparison ${ }^{3}$ between seeing limited PSF (left), tip-tilt PSF (middle) for $D / r_{0} \approx 4$, and diffraction limited PSF (right) at $\lambda=0.8 \mu \mathrm{m}$ and $\mathbf{r}_{0}=0.4 \mathrm{~m}$.

What's the size of $\mathrm{r}_{0}$ ? Giovanelli et al. ${ }^{5}$ measured a seeing of only $0.4^{\prime \prime}$ at good sites on the Chilenean Atacama plateau. The seeing is $\theta_{\mathrm{FWHM}}=0.98 \lambda / \mathrm{r}_{0}$ and the corresponding values for $\mathrm{r}_{0}$ are shown in Table $3 ; \mathrm{r}_{0}$ varies with wavelength and zenith angle according to $r_{0} \propto \lambda^{6 / 5}(\cos \zeta)^{3 / 5}$ and $\theta^{\prime}=\theta_{0}\left[\lambda_{0} / \lambda^{\prime}\right]^{-0.2}$. Obviously, one would like to concentrate the survey on areas close to zenith and image at wavelengths as long as possible. The latter requirement, fortunately, is consistent with the desire to reach $\mathrm{SNe}$ at higher redshift where the peak emission is shifted into the I-band (see Table 1). From Table 3 we adopt a mean value for $\mathrm{r}_{0}$ of $0.35 \mathrm{~m}$ at I-band. According to $\mathrm{D} / \mathrm{r}_{0}=4$ the optimum aperture size would be $1.4 \mathrm{~m}$. For sites with inferior seeing those numbers scale accordingly.

\begin{tabular}{|l|c|c|c|}
\hline & $\mathrm{V}[0.55 \mu \mathrm{m}]$ & $\mathrm{R}[0.64 \mu \mathrm{m}]$ & $\mathrm{I}[0.79 \mu \mathrm{m}]$ \\
\hline $\mathrm{r}_{0} @$ zenith & $0.25 \mathrm{~m}$ & $0.30 \mathrm{~m}$ & $0.39 \mathrm{~m}$ \\
\hline $\mathrm{r}_{0} @ 30^{\circ}$ & $0.23 \mathrm{~m}$ & $0.28 \mathrm{~m}$ & $0.36 \mathrm{~m}$ \\
\hline $\mathrm{r}_{0} @ 60^{\circ}$ & $0.16 \mathrm{~m}$ & $0.20 \mathrm{~m}$ & $0.26 \mathrm{~m}$ \\
\hline
\end{tabular}

Table 3: Values for $\mathbf{r}_{0}$ as a function of wavelength and zenith distance for an optical (V-band) seeing of $0.4^{\prime \prime}$.

\section{THE BASIC CONCEPT}

Figure 3 illustrates the basic idea. Consider two different tip-tilt "beams", i.e. columns through the atmosphere of diameter D. On average, the isoplanatic tip-tilt patch is approximately $60^{\prime \prime}$ in size. Looking at a faint guide star through one column of the atmosphere one could correct a $60^{\prime \prime}$ wide field using tip-tilt. The same is true for the adjacent "beam", which needs independent tip-tilt correction. However, a significant portion of the image motion 
occurs in turbulence layers close to the telescope and is common to both "beams". Hence we propose and describe a system with two main components:

1. a tip-tilt secondary mirror conjugated to low altitude $H$ where the beams overlap for correction of overall tip-tilt.

2. an "array" of tip-tilt elements conjugated to the height $h$ where the "beams" separate. For a $1.6 \mathrm{~m}$ aperture and a $60^{\prime \prime}$ wide "beam" that corresponds to a height of about $5500 \mathrm{~m}$ above ground.

The correction of the high-altitude component can be done with a deformable mirror (DM), similar to classical AO systems. However, while in a classical AO system the individual

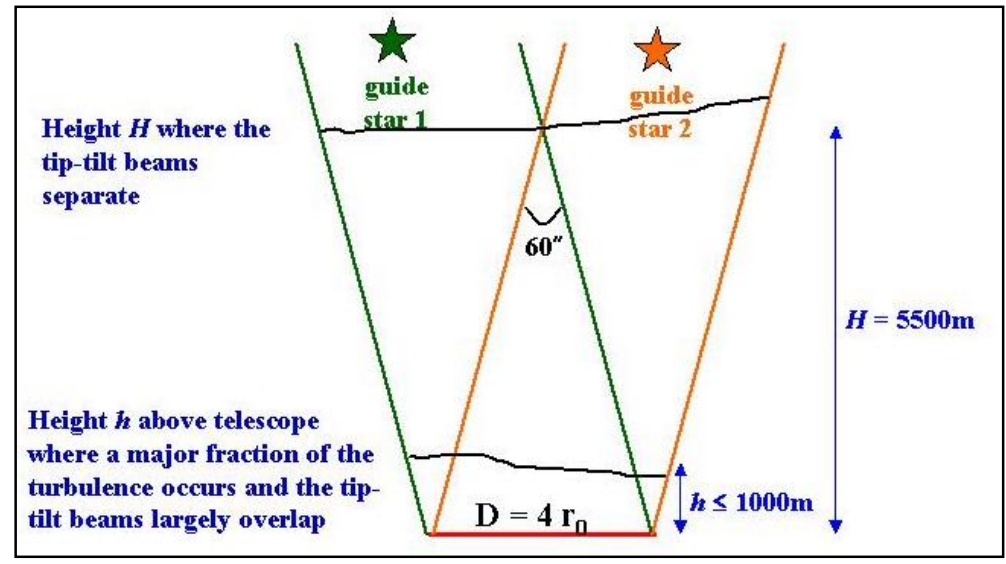

Figure 3: The basic idea. See main text for details. actuators of the DM correct small portion within one subaperture, the role of the actuators here is to provide only tip-tilt correction but over the full aperture, and each actuator corresponds to one "beam" of diameter D on the sky. Now one can basically assemble a large number of adjacent "beams" producing a wide FOV over which tip-tilt is corrected. Figure 4 shows the setup for only 4 beams, but there is no a priori technical limitation to the field size of this concept.

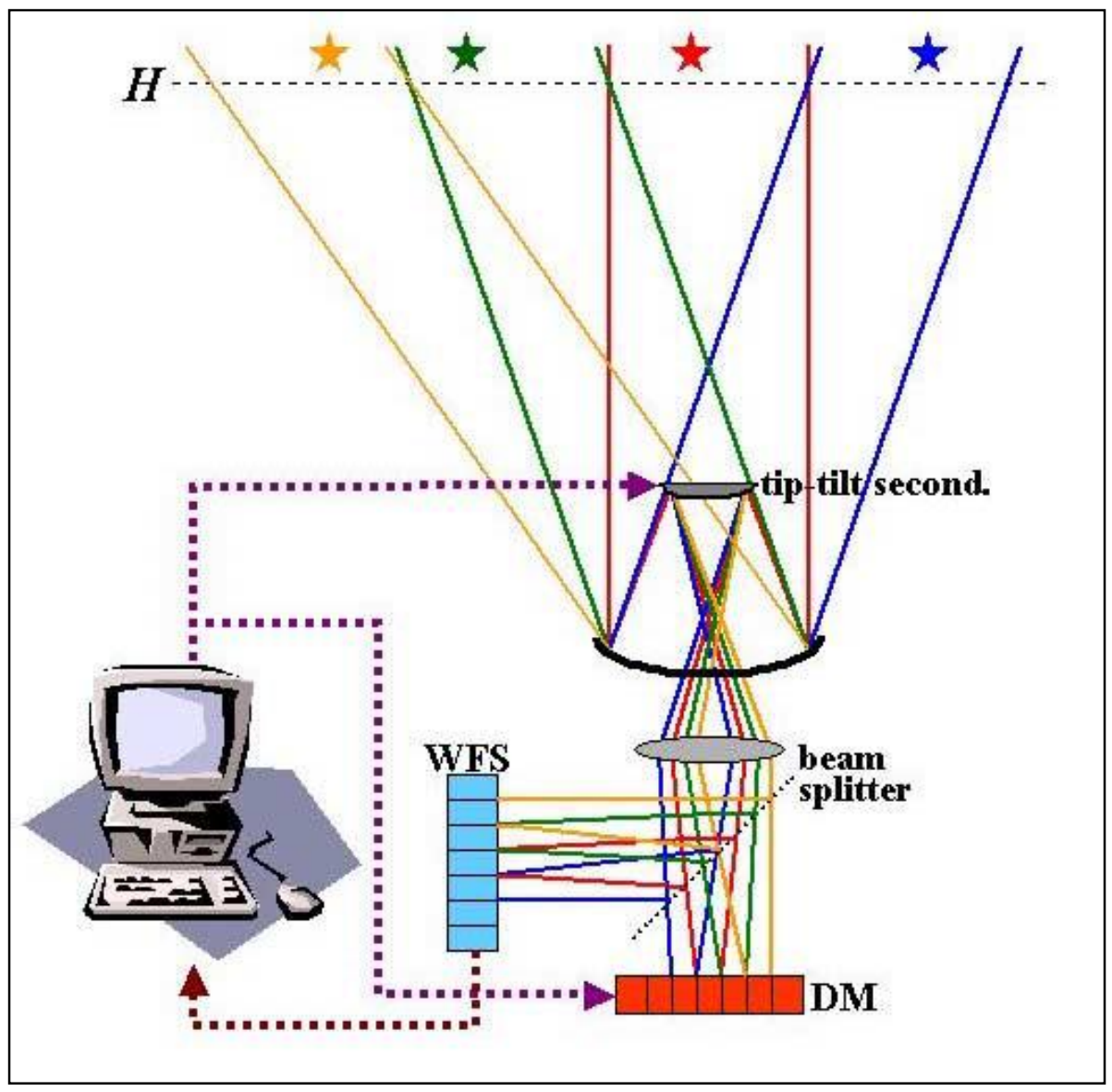

Figure 4: The basic concept illustrated for 4 adjacent tip-tilt corrected "beams". See main text for details. 


\section{WHY NOT COMPENSATE FOR THE IMAGE MOTION IN THE FOCAL PLANE?}

What the tip-tilt mirror basically does is to stabilize the image motion in the focal plane. Instead one could think of alternative ways to achieve the same goals, which were also discussed at this conference, the latter one being incorporated in the PanSSTARRS concept ${ }^{6,7}$ :

1. Apply shift-and-add techniques: the detectors are read very frequently so that the image motion gets resolved in time, the image peak (brightest speckle) is located and the images are aligned before co-adding (post processing). However, even with the most recent CCDs reading large format arrays at high bandwidth comes with high read noise and limits the sensitivity of the observations to a magnitude much brighter than required to observe faint supernovae.

2. Use orthogonal charge transfer arrays (OTA) to locate the position of the centroid (read a subportion of the array very fast); the charges in the surrounding area of the array will be shifted accordingly while still integrating photons. Compared to 1., one trades in the read noise for the much smaller charge transfer noise. However, cosmetic imperfections get smeared out over larger areas (at least the size of the seeing disk) and flatfielding will become difficult. This technique is also limited to specific focal plane CCDs (OTAs). An elegant variation of this technique uses the new generation of infrared CMOS detectors. However, the total costs to cover a reasonably large area in the focal plane are prohibitive $(\approx \$ 25 \mathrm{M}$ for $0.35 \times$ $\left.0.35 \mathrm{deg}^{2}\right)$.

Both of these techniques underlie the same limitation as our proposed approach (e.g., guide star density, limiting magnitude, isokinetic patch size, ...). However, our approach allows a much more general and flexible utilization of the focal with many significant advantages:

- Since the correction of the tip-tilt is done before the photons reach the focal plane, visitor instruments can be used.

- The science camera may use narrowband filters or dispersive elements for spectroscopy which cannot be used if the tip-tilt sensing is done in the focal plane unless one accepts a dramatically reduced sky coverage.

- The geometry (configuration) is not coupled to scales of the isokinetic patch, so any combination of pixel size and array format can be used.

- The independence of the focal plane from the sensing process allows to quickly response to new array developments that show either better performance (optimized coatings, higher DQE, larger formats, lower read noise), or simply cheaper devices.

\section{KEY COMPONENTS I: DEFORMABLE MIRROR AND WAVEFRONT SENSOR}

Like in a conventional AO system the information on the motion of the guide star is obtained from a wavefront sensor (WFS) camera, evaluated by a computer in real-time and then the information is used to control the DM (see Figure 4 for illustration). The DM must be an integral part of the common path while the light used by the WFS camera can be separated out of the common path using a beam splitter; the remaining light is then fed into the science camera. Although this general setup is very common amongst classical AO systems a several specific issues are of relevance to this system. In the following discussion we assume a FOV of $0.35 \times 0.35 \mathrm{deg}^{2}$ as a working number, although there is no hard technical limit to the field size.

- A FOV of $0.35 \times 0.35 \mathrm{deg}^{2}$ would require an array of about $21 \times 21$ actuators. Commercial devices with even a larger number of degrees of freedom exist and complexity, also in terms of computing power, is not a concern.

- Each control element (actuator) requires a maximum stroke of $15-20 \mu \mathrm{m}$ (including some safety margin for worse seeing). This is considerably more than what currently available devices with continuous face sheet mirrors yield. The reason for requiring a larger stroke is simply that the image motion now needs to be corrected over the full telescope aperture instead of a small subaperture. 
- A possible solution to the above stroke problem might be segmented MEMS DM. They have usually been avoided in classical AO systems for two reasons: (i) the gaps between segments radiate thermal emission, an undesired source of background in the infrared, and (ii) diffraction at the segment edges creates a high order structure of the PSF that makes high contrast imaging of faint companions difficult. However, none of these issues would be a concern in our applications an hence segmented MEMS DMs could be of interest.

- Ideally, the WFS chip would only need $21 \times 21$ "quad cells" to correct the image motion in $21 \times 21$ more or less independent "beams". Unfortunately, the position of the tip-tilt guide $\operatorname{star(s)~within~each~"beam"~is~not~a~priori~}$ known and varies from "beam" to "beam". Hence, each "quad cell" needs to cover the entire "beam" at a pixel resolution sufficient to determine centroid motion at the sub-arcsecond level.

- A pixel scale of $0.25^{\prime \prime} /$ pixel (certainly not an overestimate for accurate centroiding) would require a $5 \mathrm{k} \times 5 \mathrm{k}$ WFS chip of which the vast majority of pixels would be "unused" most of the time (depending on the guide star configuration on the sky). Since guide stars with sufficient sky coverage are faint, high quality WFS chips are required with $\ll 10 \mathrm{e}^{-}$read noise at typically $100 \mathrm{~Hz}$ readout frequency. Such performance has been demonstrated for smaller $128 \times 128$ pixel CCDs (up to $521 \times 512$ arrays), but $5 \mathrm{k} \times 5 \mathrm{k}$ arrays do not yet exist.

- More sophisticated methods are needed to solve the problems described above. Those include: (i) A micro-mirror array to steer the brightest guide star within each $60^{\prime \prime} \times 60^{\prime \prime}$ beam onto a much smaller portion of the WFS chip. (ii) A fish-eye lens sampled with a lenslet array. (iii) The usage of a coarser WFS pixel scale and multiple reference stars within each beam, some of which will fall onto pixel boundaries. (iv) A restriction to only the central portion of each beam where the guide star ought to be located anyway for best overall tip-tilt correction.

As with any AO system the quality of correction decreases with increasing angular distance to the guide star. Therefore stars closest to the center of each "beam" will provide the best average correction over the entire field. Field points further away will experience some residual jitter leading to a broadening of the PSF. This effect depends on wavelength, seeing and zenith distance. However, as shown in Figure 5 (right) it is rather small, typically in the order of less than $0.02^{\prime \prime}$.

With low read-noise CCDs stars as faint as $\mathrm{V}=17.7$ can be used as tip-tilt guide stars with a $\mathrm{S} / \mathrm{N} \approx 10$ at $100 \mathrm{~Hz}$ (see Figure 5, left). Since there are more late type than early type stars, the stellar density up to a certain magnitude increases toward longer wavelengths. The average density of stars with magnitude $\leq 18$ over the whole sky is $\approx 2500$ stars $/ \mathrm{deg}^{2}$ at V-band and $\approx 5500$ stars $/ \mathrm{deg}^{2}$ at I-band, varying with Galactic latitude ${ }^{8}$. Our requirement to find at least one star brighter than $18^{\text {th }}$ magnitude within one square arcminute translates into $3600 \mathrm{stars} / \mathrm{deg}^{2}$, a value that lies between the number densities for I- and V-band. We conclude that, at least for longer wavelengths for the WFS camera, the likelihood of finding tip-tilt guide stars is quite high, although there is no guarantee that every "beam" will have its own central guide star. For those "beams", where no sufficiently bright guide star could be found, the image will be "just" seeing limited but still potentially useful.

\section{KEY COMPONENTS II: THE FOCAL PLANE SCIENCE CAMERA}

The diffraction limited PSF on a $1.4 \mathrm{~m}$ telescope has a FWHM of $0.15^{\prime \prime}$ at I-band. Although larger pixel scales will provide a wider FOV it is not recommended to use scales larger than $0.1^{\prime \prime} /$ pixel to fully befit from the tip-tilt correction. To cover a $0.35 \times 0.35 \mathrm{deg}^{2}$ wide field, an array of $12 \mathrm{k} \times 12 \mathrm{k}$ pixels would be needed. As indicated in Table 3 and further discussed below, one would like to image at longer wavelengths than V-band.

As an example for an I-band sensitive CCD we have selected the Marconi ${ }^{10}$ CCD44-82, a $2048 \times 4096$ pixel BIP array with $15 \mu \mathrm{m}$ pixels. Hence $18 \mathrm{CCDs}$ would be needed to cover the $0.35 \times 0.35 \mathrm{deg}^{2}$ wide field, closely packaged with gaps of $1 \mathrm{~mm}$ between them (see Figure 6, left). The CCDs are available with optimized anti-reflection coating for higher sensitivity at longer wavelengths (see Figure 6, right). In addition, the CCDs are available in a version called "deep depletion" where the doping of the light sensitive layer has been tailored to maximum sensitivity at longer wavelengths. 

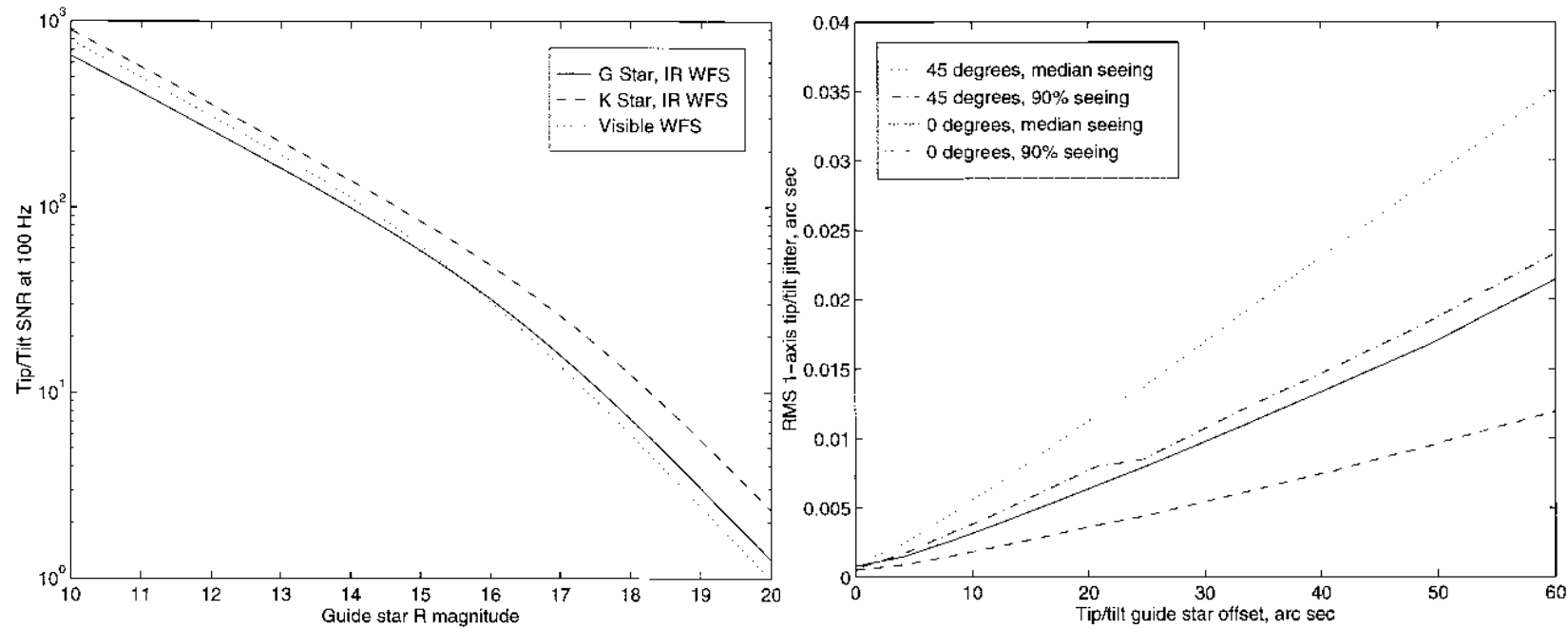

Figure 5: Left: The S/N obtained at $100 \mathrm{~Hz}$ with a low read noise $\mathrm{CCD}$ as a function of the guide star magnitude for different spectral types. Right: The RMS 1-axis jitter as a function of angular distance to the tip-tilt guide star for different seeing conditions and zenith angles?.

\section{OPEN ISSUES}

Obviously, both WFS camera and science camera would yield the best performance at I-band. On the other hand splitting the photons in the same waveband between them leads to reduced sensitivity (science camera) and smaller sky coverage (WFS camera). Hence it would be preferable to find optimum operating wavelengths for the two components that do not overlap and can be separated with a dicroïc mirror. Table 4 lists the advantages of operating WFS and science camera at a given wavelength.
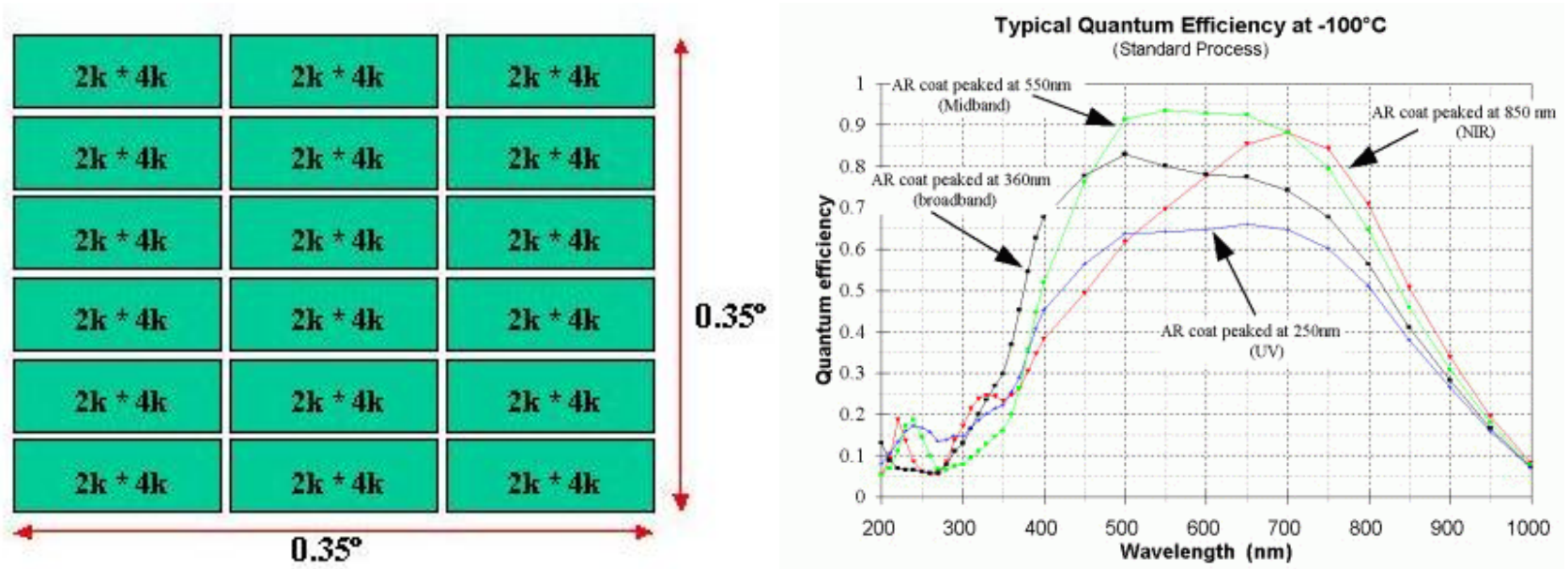

Figure 6. Left: an array of $3 \times 6$ CCDs, each $2048 \times 4096$ pixels would cover a $0.35 \times 0.35 \mathrm{deg}^{2} \mathrm{FOV}$ at $0.1^{\prime \prime}$ resolution. The physical size would be $186 \mathrm{~mm} \times 189 \mathrm{~mm}$. Right: the DQE as a function of wavelengths for different AR coatings ${ }^{10}$. 


\begin{tabular}{|c|c|c|}
\hline & WFS camera & Science camera \\
\hline short wavelength (V-band) & $\begin{array}{ll}\text { - } & \text { cheaper commercial CCDs } \\
\text { - } & \text { higher DQE } \\
\text { - } & \text { no splitting of light with SciCam }\end{array}$ & $\begin{array}{ll}- & \text { cheaper commercial CCDs } \\
- & \text { lower sky background higher } \mathrm{S} / \mathrm{N}\end{array}$ \\
\hline long wavelength (I-band) & $\begin{array}{ll}\text { - } & \text { larger sky coverage } \\
\text { - } & \text { lower bandwidth possible }\end{array}$ & 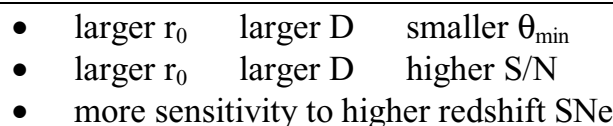 \\
\hline
\end{tabular}

Table 4: Advantages of V-and I-band as operating wavelength for WFS and science camera.

Besides the wavelength tradeoffs there are a couple of (previously mentioned) issues that need further consideration. Those include:

- The proper DM technology: can continuous face sheet DMs provide the necessary actuator stroke? Possibly segmented MEMS DMs are a better solution.

- How can focus be preserved over the full field? Segmented DMs may have the advantage to preserve focus better, in particular if adjacent "beams" experience the same wavefront tilt.

- What optics can be used to reduce the size of the WFS chip? What chip is best suited to our application?

- A complex control algorithm is needed to:

- automatically locate one or more suitable reference stars within each "beam".

- evaluate the tip-tilt information from adjacent "beams" and combine them to a smooth wavefront map without introducing sharp edges.

- reject possible bleeding from bright stars on the CCD chip.

- Are the estimates of the guide star density - a function of Galactic latitude, read noise, and spectral type - correct and sufficient?

- Variable seeing leads to variable PSF shapes and sensitivities. How much of a problem will that be in comprehensive surveys?

Although these are important issues we don't consider them to be "show stoppers". Simulations of the expected performance and feasibility studies of the technological components will be necessary.

\section{COST ESTIMATE}

For the instrument described above we list the cost estimates in Table 5.

\begin{tabular}{|l|l|}
\hline Item & Estimate \\
\hline Telescope and enclosure & $\$ 0.0 \mathrm{M}$ \\
\hline Secondary mirror modifications & $\$ 0.3 \mathrm{M}$ \\
\hline Science camera CCDs & $\$ 1.4 \mathrm{M}$ \\
\hline Science camera hard+software & $\$ 0.8 \mathrm{M}$ \\
\hline Wavefront sensor & $\$ 1.0 \mathrm{M}$ \\
\hline
\end{tabular}

\begin{tabular}{|l|l|}
\hline WFS camera optics & $\$ 1.5 \mathrm{M}$ \\
\hline Deformable mirror & $\$ 0.3 \mathrm{M}$ \\
\hline Design study, modeling & $\$ 0.4 \mathrm{M}$ \\
\hline Software development & $\$ 1.5 \mathrm{M}$ \\
\hline Computer hardware & $\$ 0.3 \mathrm{M}$ \\
\hline TOTAL & $\mathbf{\$ 7 . 5 \mathrm { M }}$ \\
\hline
\end{tabular}

Table 5: Estimated cost breakdown for the wide-field survey telescope, not including a small (1.5m class) telescope and dome itself as some existing facilities might be used. However, the costs of modifying an existing telescope (mainly the secondary mirror) are included. 


\section{SUMMARY}

1. The described telescope would enable quasi-diffraction limited searches for point sources with the high sensitivity of a $6 \mathrm{~m}$-class telescope over a large field-of-view.

2. The combination of resolution, sensitivity and FOV is unique and currently impossible with any other instrument from the ground.

3. For under $\$ 10 \mathrm{M}$ this telescope could complement vastly more expensive space missions and is likely to be of particular interest to smaller research institutes and universities.

4. The concept is very flexible and expandable:

- one unique instrument with a large FOV for under $\$ 10 \mathrm{M}$

- a downsized technology demonstrator for less than half that price

- a "network" of such telescopes, operated jointly by several universities.

1. SNAP website at http://snap.lbl.gov/

\section{REFERENCES}

2. R.J. Noll, "Phase estimates from slope sensors", J. Opt. Soc. Am., 68, 139-140, 1978

3. N. Kaiser, J.L. Tonry, \& G.A. Luppino, "A New Strategy for Deep Wide-Field High-Resolution Optical Imaging”, PASP, 112, 768-800, 2000

4. J.C. Christou, "Image Quality, Tip-tilt Correction, and Shift-and-add Infrared Imaging", PASP, 183, 1040-1048, 1991

5. R. Giovanelli et al., "The Optical/Infrared Astronomical Quality of High Atacama Sites. I. Preliminary Results of Optical Seeing", PASP, 113, 789-802, 2001

6. N. Kaiser, "Pan-STARRS: a synoptic survey telescope array", this conference \#4836-26

7. J.L. Tonry et al., "Rubber focal plane for sky surveys", this conference \#4836-35

8. C.W. Allen, “Astrophysical Quantities", University of London, The Athlone Press, 243, 1973

9. B.L. Ellerbroek \& D.W. Tyler, "Adaptive Optics Sky Coverage Calculations for the Gemini North Telescope", $P A S P, 110,165-185,1998$

10. Marconi website at http://www.marconitech.com/ccds/ 